\title{
Psychiatric sequelae of organophosphorous poisoning: a case study and review of the literature
}

\author{
R. H. Bradwell \\ Department of Psychiatry, Doncaster Royal Infirmary, Doncaster, UK \\ Correspondence to: Department of Psychiatry, Burnley General Hospital, Casterton \\ Avenue, Burnley BB10 2PQ, UK
}

\begin{abstract}
This is the case of a young farm worker presenting with episodes of acute organic psychosis superimposed on a state of chronic anergy and hypersomnia. It is suggested that he developed an encephalopathic illness presenting with an organic bipolar affective disorder as a result of organophosphate exposure. In proposing this aetiology, an hypothesis is developed which links clinical observations and investigative results with research findings in relation to organophosphorus compounds and neuropharmacology.
\end{abstract}

Keywords: Hypersensitivity - Lithium - Neurotoxicity - Organic bipolar affective disorder - Organophosphorous Phosphoinositide

\section{INTRODUCTION}

The organophosphorus (OP) compounds are complex esters of phosphoric acid or its derivatives (Heath, 1961) widely used in industry (Hamilton and Hardy, 1974) and agriculture (Clarke and Clarke, 1967; Abou-Donia, 1980). The potential for exposure is relatively great (Stuart and Oehme, 1982) and OPinduced delayed neurotoxicity (OPIDN) has been a recognized clinical syndrome for over 50 years. The pathophysiology lies in a distinctive biochemical lesion of the nerve axon caused by phosphorylation of a specific protein receptor. A delayed reaction over 1-3 weeks produces the classic OPIDN symptomatology of ataxia grading to paraplegia and ultimately death, of which more than 40000 cases have been documented between 1899 and 1989 (AbouDonia and Lapadula, 1990).

The case described here is distinct from the classical syndrome of OPIDN. There is increasing evidence that OP neurotoxicity may produce behavioural and physiological abnormalities at dosage levels well below those resulting in frank OPIDN (Redhead, 1968; Metcalf, 1982). The first published report described 16 cases and described two main reaction types following chronic exposure to OP compounds: schizophreniform and depressive (Gershon and Shaw, 1961); follow-up of these cases showed that almost all recovered within 12 months. The authors were subsequently criticized for drawing premature conclu-

(C) 1994 Rapid Communications of Oxford Ltd sions (Barnes, 1961; Bidstrop, 1961). Such psychiatric sequelae have since been described by others (Levin et al., 1976; Merrill and Mihm, 1982; Rosenthal and Cameron, 1991).

This is the case of a young farm worker presenting with episodes of acute psychosis superimposed on a state of chronic anergy, hypersomnia and loss of interest.

\section{CASE HISTORY}

In 1985 the patient was a 21 year old single man living on the family farm. His birth history and childhood were unremarkable. He left school at the age of 16 to work on another farm. He was described by his family as a quiet, shy and hard-working man. There was no history of substance misuse. His mother had suffered a postnatal depression and in 1991, a prolonged adjustment disorder with depression and anxiety following treatment for carcinoma of the breast and sudden death of her husband due to myocardial infarction. His uncle had also received treatment for a single episode of depressive neurosis.

Between 1982 and 1985 the patient had been spraying weedkillers for 3-4 days each April and September, and also pesticides, mainly demeton- $S$-methyl (Duratox) each June and aphid spraying each November. In June 1985, he developed a cold but continued 
working, spraying 12 hours a day without mask or gloves. Between 1 and 2 weeks later he noticed red blisters over his arms. At the same time he was becoming lethargic and taking increasingly to his bed. After 6 weeks his hypersomnia amounted to 15 hours sleep a day with loss of interest, paraesthesiae in his arms, bouts of sweating and frequent nonspecific headaches.

On 12 August 1985 a consultant physician noted a healing rash consisting of erythematous macules over his forearms with scarring and pigmentation. He concluded that this, together with the aforementioned symptoms, was probably due to OP poisoning which should resolve spontaneously. On the same day his brother discovered a noose in the barn. The patient was described to a consultant psychiatrist as being depressed and lethargic during the day but to have had three disturbed nights when he was agitated, disorientated and appearing to be experiencing visual hallucinations. At a subsequent interview with the psychiatrist he was withdrawn and was reluctant to discuss his experiences. However, he was not considered to be psychotic. His family reported gradual recovery over the next 4 months with 2 weeks of "normality" over Christmas before be became "excitable and childish". On 28 February 1986 he drank two pints of beer and then became tremulous, unwell and vomited. He was awake for most of the night, disturbed and noisy. For the next 48 hours he was sleepless, behaving irrationally, rambling incoherently, reciting nursery rhymes and appearing to be suffering from visual hallucinations.

On admission to Doncaster psychiatric unit he was restless, disruptive and distractible, suddenly bursting into laughter or song. He was preoccupied with names, numbers, colours, rhymes and songs. He felt "talkative and happy" and said "I am King Louis the I, II or X, the seventh son of the seventh son". His family thought that he had been experiencing visual hallucinations. He would not cooperate with a mental state examination and seemed without insight. Physical examination revealed no abnormality; blood investigations (FBC, urea and electrolytes, Lft's Tft's, blood glucose, serum cholinesterase, auto-antibodies) were all within normal ranges except for a minor elevation of AST.

He settled and slept but on awakening was overactive, with grandiose and persecutory delusions and was experiencing visual hallucinations (seeing snakes in his room). Administration of oral chlorpromazine $100 \mathrm{mg}$ followed by halolperidol $20 \mathrm{mg}$ produced severe dystonia which responded to procyclidine $10 \mathrm{mg}$ intramuscularly. He was treated with chlorpromazine to $500 \mathrm{mg}$ o.d. and lithium increasing to
$1250 \mathrm{mg}$ daily, producing a 12 hour serum level of $0.57 \mathrm{mmol} / 1$ on 5 March 1986.

His orientation, concentration and mood improved but on 17 March lithium was omitted following vomiting. Its reintroduction on 20 March again caused vomiting. After further omission his mood again became elated and he became sexually disinhibited. An EEG on 21 March showed "a mild excess of background slow activity and occasional bursts of higher voltage rhythmic and sharper activity, particularly during overbreathing." Lithium was restarted at $500 \mathrm{mg}$ o.d. and after consultation with a professor in neuropharmacology sulpiride $50 \mathrm{mg}$ o.d. was started. Four days later he was tired, hypersomnalent but euthymic. Day leave resulted in him and his family requesting discharge from hospital on 2 April 1986. He stopped taking his medication. He continued to be severely lethargic, feeling tired and sleepy even after mild exertion. A repeat EEG on June 1986 was reported as "while remaining diffusely abnormal shows a substantial improvement."

In late June 1986 a consultant neurologist found him to be quiet and withdrawn but with no specific neurological abnormality on examination. Later the same day he drank two pints of beer following which he slept until $14.00 \mathrm{~h}$ the next day. He rose, helped his father on the farm but remarked on the smell of pesticide from a neighbouring farm. That night his sleep was disrupted and he seemed agitated. He was readmitted to hospital on 3 July 1986. He was overactive and irritable with the paranoid delusion that radiation could make people believe that they were in two places at the same time. Lithium $250 \mathrm{mg}$ b.d. was prescribed with $1 \mathrm{mg}$ clonazepam t.d.s., for its antimanic properties. His mood became labile, he remained restless and manifested pressure of thought despite increasing the clonazepam to $4 \mathrm{mg}$ q.d.s. He required detention under the Mental Health Act after ransacking his room.

He improved gradually but then 2 weeks later, on 16 July 1986, was found hiding in the toilet in a tearful state, saying that he had heard his uncle's voice telling him to mutilate himself and saying "you're going to be shot dead". The clonazepam and chlorpromazine were withdrawn and lithium increased to $1250 \mathrm{mg}$ daily. On 21 July, a week later, he was miserable and oversedated, believing that his thoughts were controlled by the hospital manager, that people could read his lips, and experiencing ideas of reference in relation to the television.

He improved but refused to continue lithium after vomiting twice on $29 \mathrm{July}$ (serum Li $0.64 \mathrm{mmol} / 1$ ). He left hospital at his request on 5 August and appeared reasonably well although his mother consid- 


\begin{tabular}{|c|c|c|c|c|c|c|c|c|c|c|c|c|}
\hline & Jan & Feb & Mar & Apr & May & June & July & Aug & Sept & Oct & Nov & Dec \\
\hline 1985 & & & & & & spray & & & & & & \\
\hline 1986 & & & EEG 1 & & & EEG 2 & spray & & & & & \\
\hline 1987 & & & EEG 3 & $\emptyset$ & & & & & & & & \\
\hline 1988 & & & & & & & & & & & & \\
\hline 1989 & & & & & & & & & 0 & & & \\
\hline 1990 & & & & & & & & & & & & \\
\hline 1991 & & & & & & & & & & & & \\
\hline 1992 & & & & & & & & & $\emptyset$ & & & \\
\hline 1993 & & & & & & & & & & & & \\
\hline
\end{tabular}
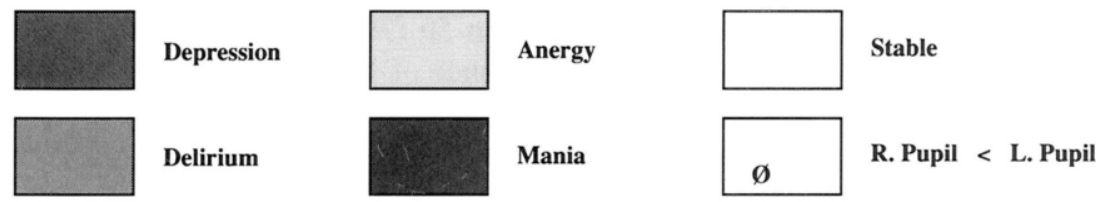

FIG. 1. Summary of the course of the illness.

ered him to be easily agitated and lethargic. On 13 January he continued to complain of lethargy and a tendency for his hands to lose colour and feel cold. His mother had noticed inequality of his pupils.

By 7 April 1987 the only abnormalities reported were loss of interest and hypersomnia. A third EEG on 8 March was reported "the trend of electro-physiological improvement continues". Computed tomography (CT) head scan showed "a general tendency to widening of extra-cerebral CSF spaces, which is probably outside the normal range of variation." An assessment by the consultant neurologist concluded "Clinical and investigative evidence indicates that he has had a severe encephalopathic illness and cyclical behaviour change which are very probably secondary to imbalance in cerebral neurotransmission caused by poisoning by organophosphorus insecticides either acutely or repeatedly."

For 2 years he remained stable, returning to work. In June 1989 whilst driving a combine harvester he lost confidence and was irritable. He developed anergy and stopped work. By September he was miserable, anhedonic, anorexic and had lost two stone in weight. He noticed that for 3 weeks his hands felt cold. On 14 September 1989 a rash was noted over the dorsal aspects of his hands, forearms and back, and the right pupil was smaller in diameter than the left. He improved spontaneously and was better by June 1990 .

On 31 July 1990 there was evidence of a hypomanic mood swing. He was readmitted to hospital under an emergency hospital order under the care of another consultant psychiatrist. He was treated with lithium $500 \mathrm{mg}$ b.d. and sulpiride $400 \mathrm{mg}$ b.d. After 2 weeks his medication was changed to lithium plus carbamazepine.

By 10 October 1990 (when serum carbamazepine $=23 \mathrm{mmol} / 1$ and serum lithium $=0.2 \mathrm{mmol} / \mathrm{l}$ ) he was recovered sufficiently to be discharged from hospital but was again lethargic. He discontinued medication in January 1991 and has continued without further mood swings to date despite the death of his father and the stresses of a court case for compensation for industrial injury due to poisoning by OP pesticides. This was eventually settled out of court for a sum which when invested would yield interest equivalent to his earnings.

Figure 1 is a diagrammatic representation of the course of the patient's illness. 


\section{DISCUSSION}

OP neurotoxicity has long been attributed to anticholinesterase activity in the neuromuscular system (Koelle and Gilman, 1946). Over subsequent years a phosphorylation site has been identified in the brain and spinal cord which is bound by the known neurotoxic OP esters but not by the non-neurotoxic OP esters (Johnson, 1969a), this site subsequently being shown to be an esterase. Further studies led to the conclusion that initial or acute neurotoxicity resulted from OP binding to and then phosphorylation of the neurotoxic esterase (NTE) with the charged group on the NTE then altering membrane function, leading to a delayed neurotoxicity (8-21 days), (Johnson, 1974). However these OP agents should not be considered solely as anticholinesterases: they are likely to have a multitoxic effect in which their inhibitory effect interacts simultaneously with adrenergic, dopaminergic and cholinergic sites. The acetylcholine released during the acute toxicity may lead to increased release of biogenic amines, and increased levels of these would be maintained because of monoamine oxidase (MAO) inhibition by the OP compounds. An excess of biogenic amines in itself is dangerous if unchecked (E1 Sebae et al., 1977).

Fatehyab et al. (1979) assayed neurotransmitters in rat brains after administration of dichlorvos (an OP compound) and found significant alterations in the levels of dopamine, noradrenaline and serotonin (5-HT). They quoted from Chase (1974): "a considerable body of anatomical, pharmacological and physiological evidence links central serotonergic mechanisms to the pathophysiology of human ills ranging from schizophrenia to Parkinsonism." Thus a link is forged between OP effects in the brain and changes in neurotransmitter mechanisms known to be important in the control of mood and behaviour, the functional balance between acetylcholine and dopamine being long established: dopamine excess producing psychosis and cholinergic deficiency cognitive impairment (Janowsky et al., 1972).

It may be that the patient's initial psychosis resulted from OP-induced malfunction of the cholinergic neuromodulatory system with consequent excess of acetylcholine in cortical and extrapyramidal systems with a dopaminergic overcompensation excess in the limbic system which produced a clinical picture of delirium (which met DSM-III-R criteria; American Psychiatric Association, 1987) but with the presence of several typical features of psychotic mania. After every episode of acute illness there was a prolonged period of anergy and hypersomnia which appeared similar to the depressive phase of bipolar illness. However the clinical features together with the subsequent course of the illness and evidence of encephalopathic damage are in keeping with an organic cause. The evidence of pupiliary inequality occurring in 1986, 1987, 1989 and 1992 raises the possibility of re-exposure to OP compounds (Grob and Harvey, 1953), or could lend weight to a hypothesis of long-term changes in the cholinergic neuromodulatory system leading to organic mood disorder but in this case bipolar in nature. Cornelius et al. (1953) have described the clinical features of patients with organic mood syndrome-depressed type (OMSD). Larsen and Richardson (1988) have delineated "organic" mania but organic bipolar affective disorder is a rarely reported phenomenon. To the author's knowledge this is the first reported case of organic bipolar affective disorder following OP poisoning.

It is possible that others exposed to OP compounds have suffered similarly. Metcalf and Holmes (1969) reported on 50 OP exposed workers and described a $45 \%$ incidence of lethargy, fatiguability, loss of interest, drowsiness and narcoleptic-like symptoms. EEGs typically showed random slowing, slow wave bursts after overbreathing and irregular theta activity. They saw this as a persistent, gradually declining but fairly specific EEG sign sensitively indicating CNS impairment. Duffy and Burchfiel (1980) thought the slow resolution of EEG abnormalities may be due to low tissue cholinesterase levels and resultant acetylcholine excess at exposure producing long-term changes in post-synaptic receptors which render them more sensitive to endogenous acetylcholine. Other studies report EEG abnormalities in OP-exposed workers. In 1989 Bernad reviewed reports relating to "EEG and pesticides". In one controlled study, 14 of the OP-exposed workers also had convulsions; these subjects all had specific EEG abnormalities consisting of bilateral synchronous theta wave activity and occasional bilateral synchronous spike and wave complexes. These specific patterns tended to return to normal within 6 months after removal from the chemical intoxicant (Hoogendam et al., 1962). Two other EEG studies involving OP-exposed workers showed an excess of slow waves, spike activity and paroxysmal bursts of delta wave abnormalities (Gupta, 1975) and bitemporal sharp waves sometimes associated with anterior slow activity (Mayersdorf and Israeli, 1974). Another report of six cases described diffuse abnormalities rather than specific findings. Thus the EEG abnormalities reported in this case are consistent with those reported in the literature to date, including the tendency to resolve over time, after exposure to the pesticide. 
It has previously been suggested that patients suffering from endogenous depression are more sensitive to the effects of acetylcholine (Dilsaver, 1986) and in a recent case report Rosenthal and Cameron (1991) suggested that their patient had an exaggerated sensitivity to the effects of an OP pesticide which resulted in recurrent depressive episodes. Mutch et al. (1992) report that variations in the activity of enzymes involved in OP toxicity could have profound effects on susceptibility to OP toxicity.

It was difficult in the present complex case to assess response to treatment but it is of interest that lithium was so consistently effective at low serum levels whilst being poorly tolerated. The action of lithium is usually regarded as "normalizing", i.e. preventing excessive mood swings away from euthymia. Baraban et al. (1989) hypothesize that action on a second messenger system could be an explanation. They state that second messenger systems are triggered by neurotransmitter receptor stimulation. The transmitter receptor complex then interacts with a GTP binding protein (G-protein). Both the cAMP system and the phosphoinositide cycle work through G-proteins and display considerable heterogeneity in the individual molecular components so that subtle effects can be achieved in different parts of the brain. In addition there is evidence that cAMP formed in response to the neurotransmitter action may modulate the actions of transmitters that act through the phosphoinositide system.

Katz and Marquis (1992) have studied low level chronic exposure to paraoxon (on OP compound) and obtained results which suggest that paraoxon caused a time-dependent increase in the accumulation of inositol phosphates and that it can modulate signal transduction by indirect activation of muscarinic receptors as well as by acting at a distal site along the pathway.

In the phosphoinositide system receptor stimulation activates a phospholipid $\mathrm{C}$ enzyme which triggers the breakdown of phosdphatidylinositol-bis-phosphate $\left(\mathrm{PIP}_{2}\right)$ to inositol trisphosphate $\left(\mathrm{IP}_{3}\right)$ and diacylglycerol (DAG). Both influence cellular function in major ways. DAG activates protein kinase $C$ which is a phosphorylating enzyme. One of its substrates is acetylcholine. In addition, protein kinase $\mathrm{C}$ has feedback effects on several aspects of the phosphoinositide system. It has been shown to have a direct role in neurotransmission including blocking the inhibitory action of several neurotransmitters including 5-HT (Baraban et al., 1985).

In OP toxicity, dose-response relationships generally depend on the amount and route of administration as well as the neurotoxic potential of a particular agent. Some OP esters are poorly absorbed orally, requiring multiple dosing to produce neurotoxicity (Stuart and Oehme, 1982). This together with the cumulative irreversible effects and poor understanding of the central effects of OP compounds may explain why cases could go unrecognized. Recent research however would seem to support an hypothesis, in respect of this patient, first formulated nearly 9 years ago.

The patient described in this paper may or may not have had an inherent diathasis toward affective disorder but it is suggested that he developed an organic bipolar affective disorder in relation to OP poisoning. It would have been interesting to have known whether the course of his illness would have been modified had he cooperated with long-term lithium maintenance treatment. The actions of lithium in mood disorder following OP exposure would seem worthy of further investigation since if both OP compounds and lithium are acting through the phosphoinositide system, there could be a rational basis for treatment of mood disorder following OP exposure with lithium.

Further research should be given impetus by the finding that exogenously administered enzymes (acetyl- or butyrlycholinesterase) will sequester OPs before they reach their physiological targets and a third enzyme, endogenous carboxylesterase, is known to be capable of scavenging OPs (Doctor et al., 1991), suggesting that there could be a preventative treatment for OP toxicity.

\section{REFERENCES}

Abou-Donia MB (1980b) Late acute, delayed neurotoxic and cholinergic effects of $S, S, S$-tritubyl phosphorotrithioite (Merphos) in hens. Toxicology and Applied Pharmacology, 53, 439-457.

Abou-Donia MB and Lapadula DM (1990) Mechanisms of organophosphorus ester-induced delayed neurotoxicity: type I and II. Annual Review of Pharmacology and Toxicology, 30, 405-440.

American Psychiatric Association (1987) Diagnostic and Statistical Manual of Mental Disorders, 3rd edn, revised. APA, Washington, DC.

Baraban JM, Worley PF and Snyder SH (1989) Second messenger systems and psychoactive drug action: Focus on the phosphoinositide system and lithium. American Journal of Psychiatry, 146, 1251-1260.

Barnes JM (1961) Psychiatric sequalae of chronic exposure to organophosphorus insecticides. Lancet, 2, 102-103.

Bernad PG (1989) Review: EEG and pesticides. Clinical Electroencephalography, 20, IX-X.

Bidstrup PL (1961) Psychiatric sequalae of chronic exposure to organophosphorus insecticides. Lancet, 2, 103.

Chase TN (1974) In: Advances in Neurology (Eds FH McDowell and A Barbeau), Vol. 5, p. 31. Raven Press, New York. 
Clarke EGC and Clarke ML (1967) In: Veterinary Toxicology, 3rd edn (Eds Tindall and Cassell), pp. 242-261. Balliere, London.

Cornelius JR, Fabrega H Jr, Mezzich J, Cornelius MD and Ulrich RF (1993) Characterising organic mood syndrome, depressed type. Comprehensive Psychiatry, 34, 432-440.

Dilsaver SC (1986) Cholinergic theory of depression. Brain Research Reviews, 11, 285-316.

Doctor BP, Raveh L, Wolfe AD, Maxwell DM and Ashani Y (1991) Enzymes as pre-treatment drugs for organophosphate toxicity. Neuroscience and Biobehavioural Reviews, 15, 123-128.

Duffy FH and Burchfiel JH (1980) Long term effects of the organophosphorus Sarin on EEGs in monkeys and humans. Neurotoxicology, I, 667-698.

El-Sabae AH, Soliman SA, Elamayem A and Ahmed NS (1977) Neurotoxicity of organophosphorus insecticides Leptophos and EPN. Journal of Environmental Science and Health, 12B, 269-288.

Fatehyab Ali S, Hassan M and Tariq M (1971) Levels of dopamine, norepinephrine and 5-hydroxytryptamine in different regions of rat brain and spinal cord following chronic administration of organophosphate pesticide dichlorvos. Indian Journal of Experimental Biology, 17, 424-426.

Gershon S and Shaw FH (1961) Psychiatric sequelae of chronic exposure to organophosphorus insecticides. Lancet, 1, 1371-1374.

Grob D and Harvey AM (1953) The effects and treatment of nerve gas poisoning. American Journal of Medicine, 14, 52-63.

Gupta PC (1975) Neurotoxicity of chronic chlorinated hydrocarbon insecticide poisoning: A chemical and electro-encephalographic study in man. Indian Journal of Medical Research, 63, 601-606.

Hamilton A and Hardy HL (1974) Industrial Toxicology, 3rd edn, pp. 315-317, 353-361. Public Sciences Group Inc., Acton, MA.

Heath DF (1961) Organophosphorus Poisons. Pergamon Press, New York.

Hoogendam I, Versteeg JPJ and De Vlieger M (1962) Electroencephalograms in insecticide toxicity. Archives of Environmental Health, 4, 92-100.

Janowsky DS, El-Yousef MK, Davis JM and Sekerke HJ (1972) A cholinergic adrenergic hypothesis of mania and depression. Lancet, 2, 632-635.

Johnson MK (1969) A phosphorylation site in brain and delayed neurotoxic effect of some organophosphorus compounds. Biochemical Journal, 111, 487-495.
Johnson MK (1974) The primary biochemical lesion leading to the delayed neurotoxic effects of some organophosphorus compounds. Journal of Neurochemistry, 23, 785-789.

Katz LS and Marquis JK (1992) Organophosphate induced alterations in muscarinic receptor binding and phosphinositide hydrolysis in the human SK-N-SH cell line. NeuroToxicology, 13, 365-378.

Koelle GB and Gilman A (1946) The chronic toxicity of di-isopropyl fluorophosphate (DFP) in dogs, monkeys and rats. Journal of Pharmacology and Experimental Therapeutics, 87, 435-448.

Larson EW (1988) Organic causes of mania. Mayo Clinic Proceedings, 63, 906-912.

Levin HS, Rodnitzsky RL and Mick DL (1976) Anxiety associated with exposure to organophosphate compounds. Archives of General Psychiatry, 33, 225-228.

Mayersdorf A and Israeli R (1974) Toxic effects of chlorinated hydrocarbon insecticides on the human electroencephalogram. Archives of Environmental Health, 28, 159163.

Merrill DG and Mihm FG (1982) Prolonged toxicity of organophosphorus poisoning. Critical Care Medicine, 10, 550-551.

Metcalf DR and Holmes JH (1969) EEG, psychological and neurological alterations in humans with organophosphorus exposure. Annals of the New York Academy of Sciences, 106, 357-365.

Metcalf RL (1982) Historical perspective of organophosphorus ester-induced, delayed neurotoxicity. NeuroToxicology, 3, 269-284.

Mutch E, Blain PG and Williams FM (1992) Interindividual variations in enzymes controlling organophosphate toxicity in man. Human and Experimental Toxicology, 11, 109-116.

Redhead IH (1968) Poisoning on the farm. Lancet, 1, 686688.

Rosenthal NE and Cameron CL (1991) Exaggerated sensitivity to an organophosphate pesticide. American Journal of Psychiatry, 148, 270.

Stuart LD and Oehme FW (1982) Organophosphorus delayed neurotoxicity: A neuromyelopathy of animals and man. Veterinary and Human Toxicology, 24, 107-118.

(Received 12 April 1994; accepted as revised 20 October 1994) 


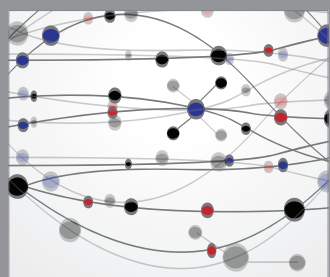

The Scientific World Journal
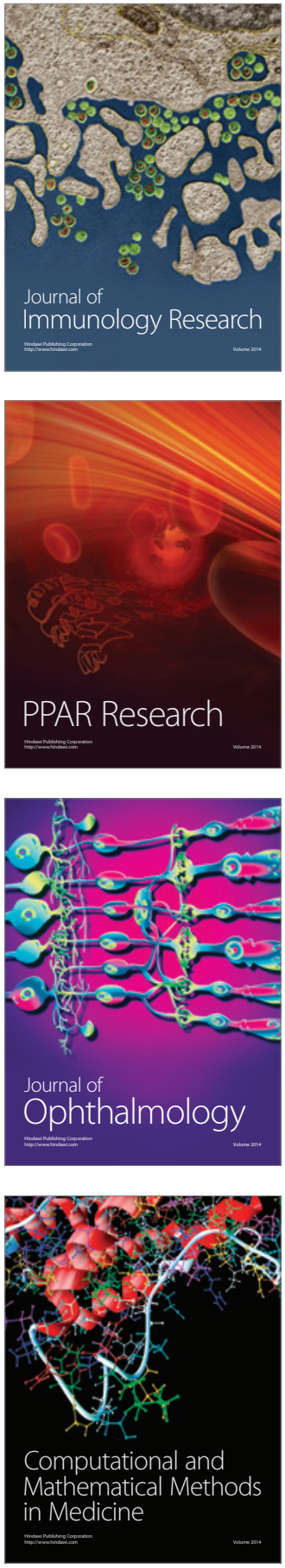

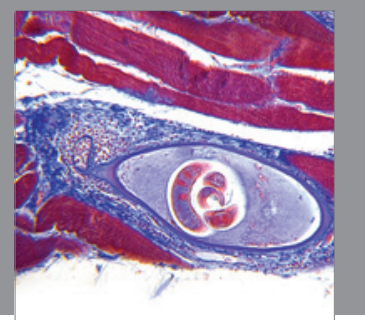

Gastroenterology

Research and Practice
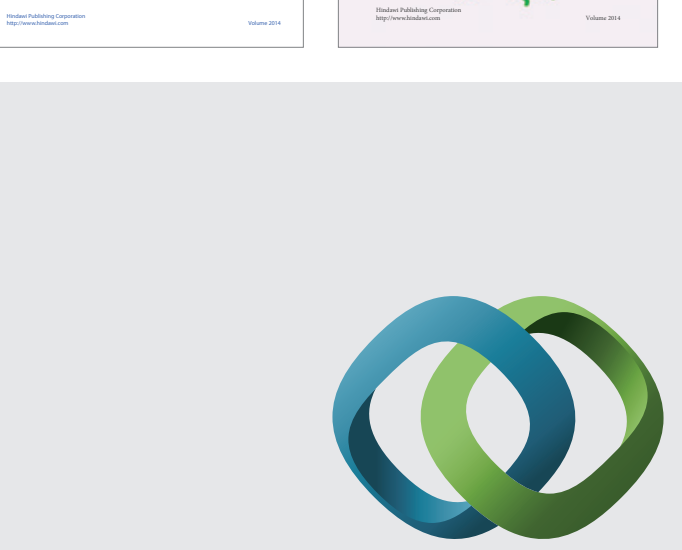

\section{Hindawi}

Submit your manuscripts at

http://www.hindawi.com
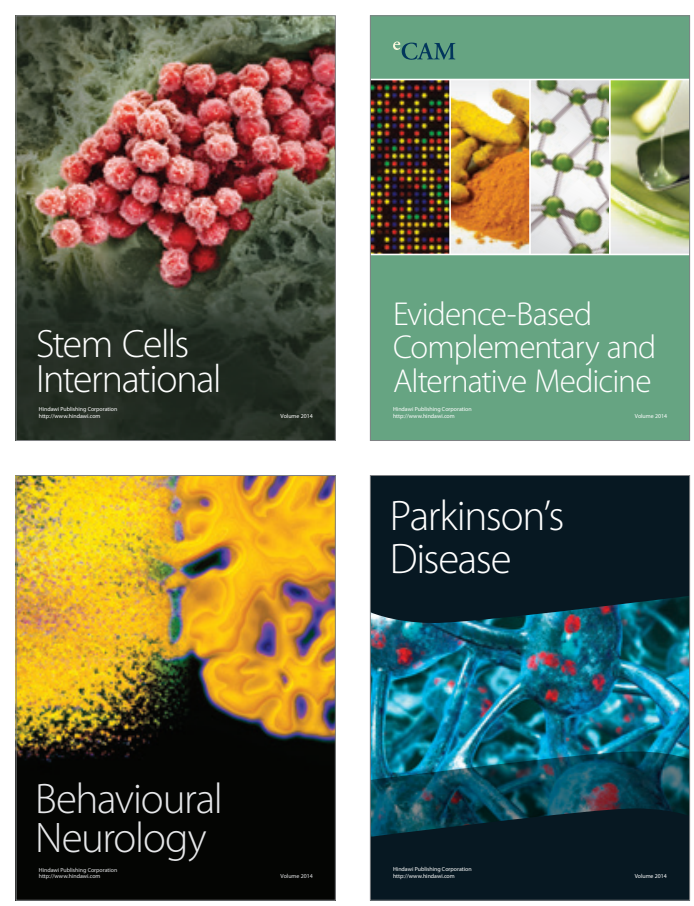

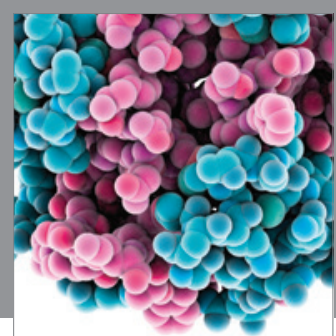

Journal of
Diabetes Research

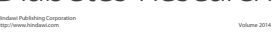

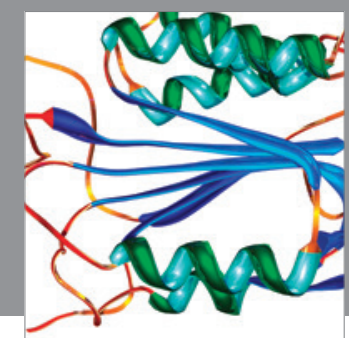

Disease Markers
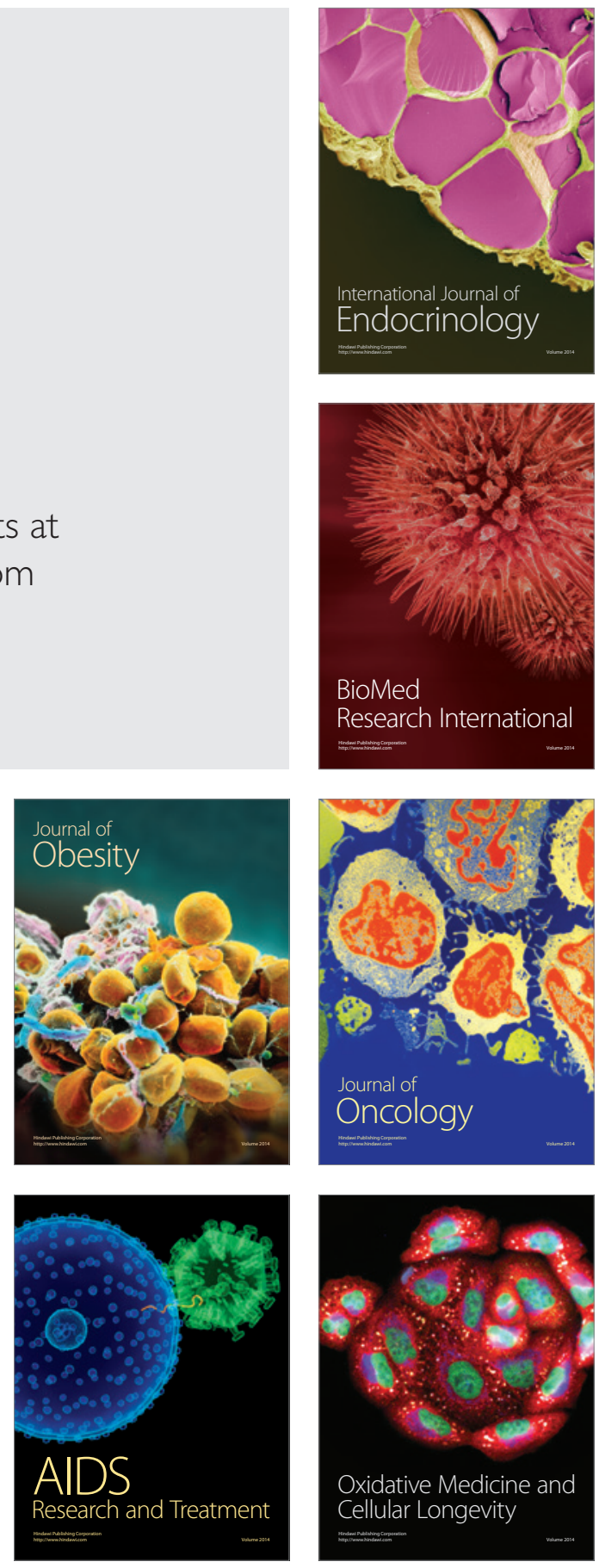